\title{
Lights and Shadows of Distal Deep Vein Thrombosis
}

Cristiano Bortoluzzi ${ }^{1 *}$, Giuseppe Camporese ${ }^{2}$, Ngoc Vo Hong ${ }^{1}$, Enrico Bernardi ${ }^{3}$, Pierpaolo Di Micco ${ }^{4}$, Raffaele Pesavento ${ }^{5}$ and Roberto Parisi $^{1}$

${ }^{1}$ SS Giovanni e Paolo Hospital of Venice, Italy

2University Hospital of Padua, Italy

${ }^{3} \mathrm{H}$ ospital of Conegliano, Treviso, Italy

${ }^{4}$ Fatebenefratelli Hospital of Naples, Italy

${ }^{5}$ University Hospital of Padua, Italy

\begin{abstract}
Distal deep venous thrombosis (DDVTs) is one of the "grey" areas of venous thromboembolism. There is a great heterogeneity in the diagnostic and therapeutic strategies between all the diagnostic centers. Studies doesn't clarify the problem so it is not clear yet if there is any advantage in diagnosing and consequentially treating all the IDDVT. The 2012 ACCP guidelines suggested clinical observation for 2 weeks over initial anticoagulation (grade 2C) in patients with acute IDDVT without severe symptoms or risk factors for extension.
\end{abstract}

\section{Introduction}

Distal deep venous thrombosis (DDVT) is one of the "grey" areas of venous thromboembolism. Usually DDVT are divided in isolated distal deep venous thrombosis and extended distal deep vein thrombosis in which there is a thrombus extension toward proximal vessels as popliteal and femoral veins. For this reason the real incidence in the world of DDVT is really difficult to be established but there is a common and historical consensus to consider DDVT less common than proximal DVT [1]. Furthermore, the appearance of asymptomatic DDVT is a further confounding factor to have a clear incidence of DDVT.

In daily clinical practice extended DDVT are considered as proximal DVT not only for a diagnostic point of view but also as from a therapeutic point of view while IDDVTs show several open issues $[1,2]$. Diagnostic methods, clinical approach, therapeutic strategies, antithrombotic regimens, methods to prevent recurrences are only the most common debated issues concerning the management of IDDVT. This review is focused to underline the most common points of discussion concerning IDDVT.

\section{Diagnostic Approach to DDVT}

Diagnostic methods of DDVT are still matter of discussion in daily clinical management in the scientific community. The diagnostic approach is different in several centers in the world as reported by several Authors, but these different methodologies for diagnosis have been found to be effective and safe and are all commonly accepted in clinical practice $[2,3]$. Symptomatic patients for calf pain usually are selected to perform a full evaluation including clinical examination, pretesting probability for DVT, d-dimer dosages and ultrasound scan with Doppler evaluation [2,3]; patients that after this screening have not all needed aspects for an IDDVT diagnosis may be invited to perform an instrumental evaluation after 1-2 weeks; moreover, usually, according to several guidelines also the choice to treat an IDDVT after a positive whole limb ultrasound scan with Doppler may be associated to a further control after 2 weeks if not associated to several aspects or clinical conditions (e.g. positive d-dimer, cancer and so on) [3].

However, the routine use of the whole limb Doppler ultrasound in clinical practice has increased the diagnosis of DDVT. The whole limb ultrasound testing has been showed particularly effective to detect asymptomatic DDVT [2-6].

\section{Risk Factors}

Strong and common risk factors for proximal DVT are recent surgery, prolonged hypomobility, pregnancy, hormonal treatment (e.g., oral contraceptive use), cancer and its related therapy. These risk factors and lower limb trauma not associated to surgery are also considered major risk factors for IDDVT. In a recent study by Donadini et al. [5] the most common acquired thrombotic risk factors in IDDVT was cancer in nearly $50 \%$ of cases and in the same study cancer was the most common risk factor associated to recurrent VTE appearing as IDDVT [7]. Thrombophilia testing is not routinely suggested after an episode of IDDVT and the presence of thrombophilia is not considered a relevant factor to establish the duration of antithrombotic treatment after a proximal DVT or distal DVT according to ACCP guidelines $[8,9]$.

Thrombophilic screening performed in a study by Kovac et al. [9] in fact, does not show any difference concerning the occurrence of IDDVT in thrombophilic vs. non-thrombophilic patients. Several other clinical conditions have been underlined as risk factor for a DVT as inflammatory bowel disease, prolonged use of several drugs, placement of central venous catheters or peripherally inserted central catheter, kidney failure, antiphospholipid syndrome but their presence is not associated to a routinely screening and prophylaxis of proximal DVT and this aspect is not available for distal DVT. Recent trauma without surgery of calf represent a frequent risk factor for IDDVT but a clear incidence of this clinical event is difficult and this aspect seem to influence also the incidence of asymptomatic IDDVT in which a recent trauma in the anamnesis is difficult to be found.

\section{DDVT Prophylaxis and Therapeutic Approach}

There are not available studies andlor guidelines concerning

*Corresponding author: Cristiano Bortoluzzi, UO Medicine, SS Giovanni e Paolo Hospital of Venice, Italy, Tel: 00393398078146; E-mail: crisbort@libero.it

Received January 10, 2018; Accepted January 25, 2018; Published January 29 2018

Citation: Bortoluzzi C, Camporese G, Hong NV, Bernardi E, Micco PD, et al. (2018) Lights and Shadows of Distal Deep Vein Thrombosis. J Blood Lymph 8 : 201. doi:10.4172/2165-7831.1000201

Copyright: (c) 2018 Bortoluzzi C, et al. This is an open-access article distributed under the terms of the Creative Commons Attribution License, which permits unrestricted use, distribution, and reproduction in any medium, provided the original author and source are credited. 
prophylaxis of IDDVT. For the exposed reasons the Literature offers different opinion concerning treatment regimens for IDDVT. Different opinion is also available concerning the type of antithrombotic drugs and dosages to treat an IDDVT

ACCP guidelines published in 2008 recommended a long-term (for at least 3 months) anticoagulant treatment for all diagnosed DVT, including calf DVT. In contrast, the 2012 ACCP guidelines suggested clinical observation for 2 weeks over initial anticoagulation (grade 2C) in patients with acute IDDVT without severe symptoms or risk factors for extension $[10,11]$. So, a thorough evaluation of risklbenefit of antithrombotic treatment for a DDVT is always suggested. Moreover, 2016 ACCP guidelines recommend to treat DDVT in a similar way and intensity as proximal DVT when further risk factors are present or symptoms are so close, although this clinical suggestion has a low grade of recommendation (2C) [11].

Concerning the type of treatment there is not specification on international guidelines concerning the type of antithrombotic drugs. For several years low molecular weight heparins (LMWHs) were considered the first line drugs involved for the treatment of IDDVT. A study involving low-risk patients showed that IDDVT treatment with nadroparin is effective in reducing venous thromboembolic events but with a particularly high risk of bleeding and concluded that for these patients clinical observation alone is an adequate approach and anticoagulant therapy is not needed [12]. There is however an earlier work by Parisi et al. [13]. which demonstrated how a treatment with low-molecular-weight heparin for 1 week at full dosage and then at half dose for other 3 weeks is safe and effective in patients with DDVT associated with transient risk factors. In a more recent study LMWHs were administered for 4-6 weeks with a significant efficacy for recurrent VTE.

However available anticoagulants useful for the treatment of IDDVT are summarized in Table 1.

\begin{tabular}{|c|c|c|c|}
\hline $\begin{array}{c}\text { Type of drug to } \\
\text { treat IDDVT }\end{array}$ & $\begin{array}{c}\text { Mentioned in } \\
\text { International } \\
\text { guidelines }\end{array}$ & $\begin{array}{c}\text { Used Randomized } \\
\text { clinical trials }\end{array}$ & $\begin{array}{c}\text { Used with real life } \\
\text { evidences }\end{array}$ \\
\hline $\begin{array}{c}\text { Low molecular } \\
\text { weight heparin }\end{array}$ & Not specifically & Yes & Yes \\
\hline Fondaparinux & Not specifically & No & Yes \\
\hline Antivitamin K drugs & Not specifically & No & Yes \\
\hline $\begin{array}{c}\text { Direct oral } \\
\text { anticoagulants }\end{array}$ & Not specifically & No & Yes \\
\hline
\end{tabular}

Table 1: Anticoagulant drugs to treat IDDVT and related scientific evidences.

Yet, because the suggestion of ACCP to treat symptomatic IDDVT as a proximal DVT also anti-vitamin $\mathrm{K}$ drugs are considered for long term treatment of a DDVT as suggested by international experts [11]. Actually based on the same guidelines also direct oral anticoagulants (DOACs) may be considered although there are not randomized clinical trials on DOACs in the treatment of IDDVT.

\section{Take Home Messages}

Calf vein thrombosis may appear as IDDVT or extended DVT.
Diagnosis of IDDVT may be on symptomatic patients or may be incidental and actually this clinical aspect represents a great dilemma to choose the correct therapeutic approach: symptomatic patients that have also underling thrombotic risk factors are selected for long term treatment. Few randomized trials are available for the treatment of IDDVT with anticoagulants in particular Low Molecular Weight Heparin while reduced data are available for fondaparinux, antivitamin $\mathrm{K}$ drug or direct oral anticoagulants. The prophylaxis of IDDVT is similar to the prophylaxis of other form of lower limb DVT although specific and addressed studies are lacking in the literature.

\section{References}

1. Cogo A, Lensing AW, Prandoni P, Hirsh J (1993) Distribution of thrombosis in patients with symptomatic deep vein thrombosis. Arch Intern Med 153: 2777 2780 .

2. Galanaud JP, Quenet S, Rivron-Guillot K, Quere I, Muñoz-Torrero JFS, et al (2009) Comparison of the clinical history of symptomatic isolated distal deepvein thrombosis vs. proximal deep vein thrombosis in 11086 patients. J Thromb Haemost 7: 2028-2034

3. Bernardi E, Camporese G, Buller HR, Siragusa S, Imberti D, et al. (2008) Serial 2-point ultrasonography plus D-dimer vs whole-leg color-coded Doppler ultrasonography for diagnosing suspected symptomatic deep vein thrombosis: a randomized controlled trial. JAMA 300: 1653-1659.

4. Galeandro AI, Quistelli G, Scicchitano P, Gesualdo M, Zito A, et al. (2012) Doppler ultrasound venous mapping of the lower limbs. Vasc Health Risk Manag 8: 59-64.

5. Donadini MP, Dentali F, Pegoraro S, Pomero F, Brignone C, et al. (2017) Longterm recurrence of venous thromboembolism after short-term treatment of symptomatic isolated distal deep vein thrombosis: A cohort study. Vasc Med 22: $518-524$

6. Righini M, Paris S, Le Gal G, Laroche JP, Perrier A, et al. (2006) Clinical relevance of distal deep vein thrombosis. Review of literature data. Thromb Haemost 95: 56-64.

7. Galanaud JP, Sevestre MA, Genty C, Kahn SR, Pernod G, et al. (2014) Incidence and predictors of venous thromboembolism recurrence after a first isolated distal deep vein thrombosis. J Thromb Haemost 12: 436-443.

8. Kovac M, Mitic G, Mikovic Z, Antonijevic N, Djordjevic V, et al. (2008) Type and location of venous thromboembolism in carriers of Factor $V$ Leiden or prothrombin G20210A mutation versus patients with no mutation. Clin Appl Thromb Hemost 16: 66-70.

9. Kearon C, AkI EA, Ornelas J, Blaivas A, Jimenez D, et al. (2016) Antithrombotic therapy for VTE disease: CHEST Guideline and Expert Panel Report. Chest 149: 315-352.

10. Kearon C, Akl EA, Comerota AJ, Prandoni P, Bounameaux H, et al. (2012) Antithrombotic Therapy for VTE disease: Antithrombotic Therapy and Prevention of Thrombosis, (9th edn.), American College of Chest Physicians Evidence-Based Clinical Practice Guidelines. Chest 141: 419-494.

11. Kearon C, Akl EA, Ornelas J, Blaivas A, Jimenez D, et al. (2016) Antithrombotic Therapy for VTE Disease: CHEST Guideline and Expert Panel Report. Chest 149: $315-352$.

12. Righini M, Galanaud JP, Guenneguez H, Brisot D, Diard A, et al. (2016) Anticoagulant therapy for symptomatic calf deep vein thrombosis (CACTUS): A randomised, double-blind, placebo-controlled trial. Lancet Haematol 3 : e556-e562.

13. Parisi R, Visonà A, Camporese G, Verlato F, Lessiani G, et al. (2009) Isolated distal deep vein thrombosis: Efficacy and safety of a protocol treatment. Treatment of Isolated calf Thrombosis (TICT) Study. Int Angiol 28: 68-72. 